\title{
Active Filtering and VAR Control of a Cascaded H-bridge Multi-level StatCom
}

\author{
R.E. Betz \\ School of Electrical Engineering \\ and Computer Science \\ University of Newcastle \\ NSW 2308, Australia \\ Robert.Betz@newcastle.edu.au
}

\author{
T.J. Summers \\ School of Electrical Engineering \\ and Computer Science \\ University of Newcastle \\ NSW 2308, Australia \\ Terry.Summers@newcastle.edu.au
}

\author{
G. Mirzaeva \\ School of Electrical Engineering \\ and Computer Science \\ University of Newcastle \\ NSW 2308, Australia \\ Galina.Mirzaeva@newcastle.edu.au
}

\begin{abstract}
This paper investigates the control of a multi-level cascaded H-bridge STATCOM when used as a simultaneous active filter and VAR compensator. The control algorithms developed borrow heavily from control strategies used in variable speed drives, and use predictive current control and symmetrical PWM. A novel phase locked loop approach for the extraction of the harmonics and generation of the forward prediction of the harmonic reference currents is developed. Simulation results in this abstract are presented. The final paper will present experimental results as well.
\end{abstract}

Index Terms-StatCom, VAR compensation, Active Filtering, Multilevel, H-bridge.

\section{INTRODUCTION}

The Cascade H-bridge multi-level converter is becoming a popular choice for the implementation of multi-level Static Compensators (StatCom). The main reasons that this topology is being chosen for this application are:

1) A high number of levels can be implemented because the number of components scales linearly with the level number (other topologies, such as the neutral point clamped converter (NPCC) and Flying Capacitor Converter (FCC) scale quadratically);

2) it is possible to balance the capacitor voltages, even for system with many levels;

3) the modular nature of the topology makes construction and maintenance simpler;

4) the high number of levels available makes it possible to directly connect the StatCom to the grid supply up to medium distribution voltages. This eliminates the bulky, lossy and expensive transformer required with other topologies;

5) the topology is ideally suited to applications that do not involve real power. Therefore active filtering and VAR control are a naturally fit to the topology's strengths.

Throughout the remainder of this digest the acronym $\mathrm{H}$ StatCom will be used to refer to a Multi-level StatCom implemented with cascaded H-bridges.

The traditional techniques for controlling the firing of the $\mathrm{H}$ bridges in the individual phase legs use either multiple triangular carrier waves for each of the H-bridges [1], or precomputed firing angles for the bridges to implement selective harmonic elimination (SHE) [2], [3]. The firing control technique used in the algorithms that are the subject of this paper are based on the instantaneous power concept [4], [5] coupled with deadbeat (aka predictive) current control and symmetrical PWM [6], [7], both of which are exactly the same as used in many variable speed drive systems [8]. One difference in the $\mathrm{H}$ StatCom application is that the PWM generation algorithm and the capacitor voltage balancing algorithms are tightly integrated.

An advantage of this approach is that it operates in the stationary reference frame, and the control at any instant of time is dependent only on values measured or computed at that time. The dead-beat nature of the current control loops mean that their bandwidth is very high. The control calculations are numerically simple, and therefore the control technique is ideally suited for digital implementation. Furthermore, as mentioned above, it is relatively simple to incorporate a very high performance capacitor voltage balancing algorithm into the PWM generation strategy. This balancing approach uses a logic based algorithm to evenly share the leg cluster voltage (whatever it is) across the H-bridge capacitors in each leg. A second leg cluster voltage control loop keeps the cluster voltages at a set-point value [8]. This technique avoids some of the control loop interaction problems present in other strategies [9]. A disadvantage of the PWM approach is that the first significant phase harmonics are at the H-bridge switching frequency, which is not as high as obtainable with phase shifted carrier waveform based PWM approaches [5].

This paper extends previous work of the authors by implementing active filter and VAR compensation control for a $\mathrm{H}$ StatCom. The active filter approach is not using instantaneous power as the basis of the filtering, but instead is using an interesting phase locked loop structure (PLL) to isolate the objectionable harmonics to be filtered. A very important implementation detail with the approach taken is that the PLL implementation generates very accurate forward projections of the harmonic currents thereby virtually completely eliminating the effects of computational delay on the performance of the filtering algorithm. This is especially important in systems with low control rates. In addition this PLL technique is able to accurately follow, without any phase error, the changes in 


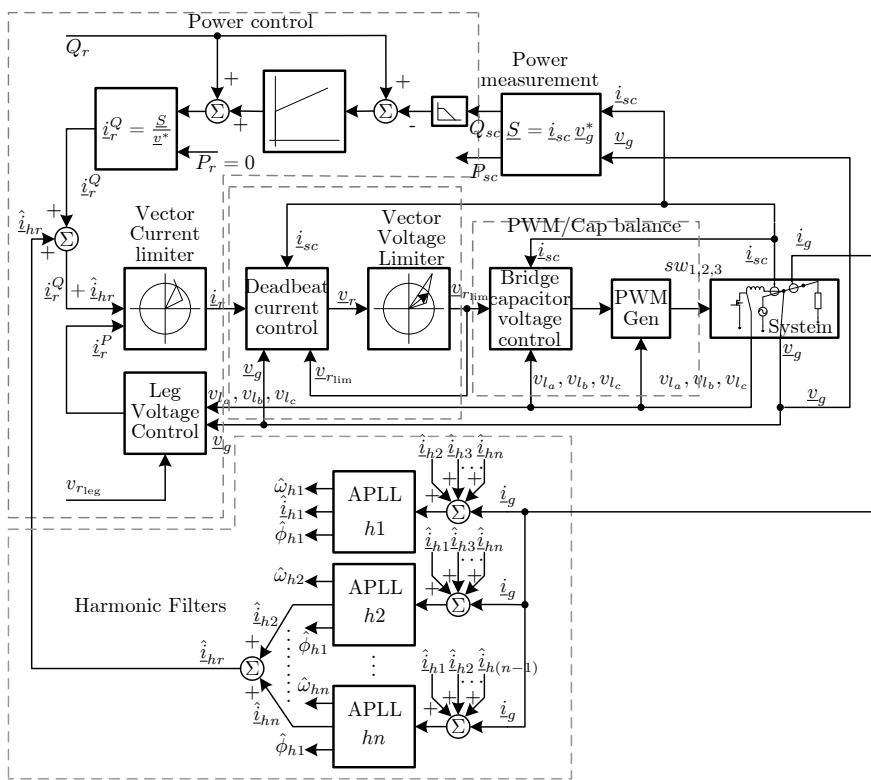

Figure 1: Block diagram of the H-StatCom control with VAR compensation and active filtering.

the frequency of the supply and the associated harmonics. A significant advantage of this technique is that the use of high accuracy PLLs means that excellent harmonic elimination can be achieved using only feed-forward of the harmonic currents. This avoids possible stability issues that can occur with harmonic feedback approaches.

\section{THE ALGORITHM STRUCTURE}

Figure 1 is a block diagram of the control algorithm. Most of the details in this figure have been presented previously [8], however there is the notable addition of a harmonic filtering section and an additional feed-forward path of the harmonic current estimates $\left(\hat{i}_{h r}\right)$ to the input of the current limiter.

The control in Figure 1 implements both traditional VAR compensation (i.e. controlling the fundamental frequency reactive power on the network), as well as simultaneously implementing active filtering. The reference VARs, $Q_{r}$, which is the desired output $Q$ for the H-StatCom, is derived from external control loops that determine this from the overall control objective of the system. This results in the desired current component $\underline{i}_{r}^{Q}$. The harmonic current required is determined from the harmonic currents being drawn by the load. The harmonic current required is then fed-forward to the current controller as $\hat{i}_{h r}$, where the "^" means that this is an estimated value.

Remark 1: Note that there is no feedback in the current algorithm with respect to the harmonics. Often controllers of this type use a combination of feed-forward and feedback in order to eliminate harmonics [9]. Whilst this leads to excellent harmonic rejection, it can also result in potential instability. If the current control and the harmonic measurement system have high accuracy then excellent performance can be achieved without feedback (as shall be shown in this paper).
It was realised that the production of a very accurate $\hat{i}_{h r}$ estimate was the crucial technical challenge for the active filter. Therefore the remainder of this paper will concentrate on a new technique for achieving this using a novel PLL structure.

\section{The Advanced Phase Locked Loop (APLL)}

The dead-beat control approach means that there is effectively a sample delay between the calculation of the control and the application of the control. Therefore for accurate control, very precise predictions are required for the system grid voltage during the next control interval (this parameter appears directly in the predictive current controller). In the case of active filtering a forward prediction of the harmonic is also required so that the current from the H-StatCom is precisely in phase with the desired harmonic. If this is not achieved, then one sample delay, especially at low to medium switching frequencies, severely compromises the performance of the active filter since it prevents the current controller from accurately producing the required harmonic current - there will be a phase shift between the desired harmonic output and the desired harmonic output. The H-StatCom, which is the subject of this research, has a control interval of $400 \mu \mathrm{sec}$. A half period of the 5 th harmonic is $2 \mathrm{msec}$, therefore the digital delay is $20 \%$ of the harmonic half cycle. If nothing was done about this there would be at least a $36^{\circ}$ phase delay in the 5 th harmonic current produced by the $\mathrm{H}$-StatCom relative to the reference. The situation is worse for the 7 th and higher harmonics.

Remark 2: The concept of separating out the harmonics is crucial to the active filtering approach undertaken in this paper. Some approaches to active filtering essentially remove the fundamental component from the distorted current or voltage waveform, and then the residual is uses as the reference. The idea is that this will remove all of the residual and only leave the fundamental. However, it is very difficult to generate a forward prediction of the residual, since, in principle, it has an unknown functional form. Consequently, this approach one works acceptably when the switching and control frequency is high, since the effects of digital delay are minimised under these conditions. However, if the harmonics are individually separated from the residual, then we do have waveforms of a known functional form, and therefore very accurate forward prediction is possible.

Other standard techniques for extracting harmonics in StatCom applications involve either bandpass filters [10], or Park transformations and high/low pass filters [5]. Both these techniques can have problems - the high Q bandpass filters produce unacceptable phase shift if the supply frequency is not exactly as expected, and the Park transformation low pass/high pass filter techniques can produce similar effects when multiple harmonics are involved. The authors have developed a technique for harmonic extraction, which also allows very accurate forward prediction of the harmonics, and is based on the use of PLLs. This concept is not new, as there is extensive literature on the subject. For example, the Karimi-Ghartemani EPLL concept [11] has been extensively researched, and other forms 
FUNDAMENTAL

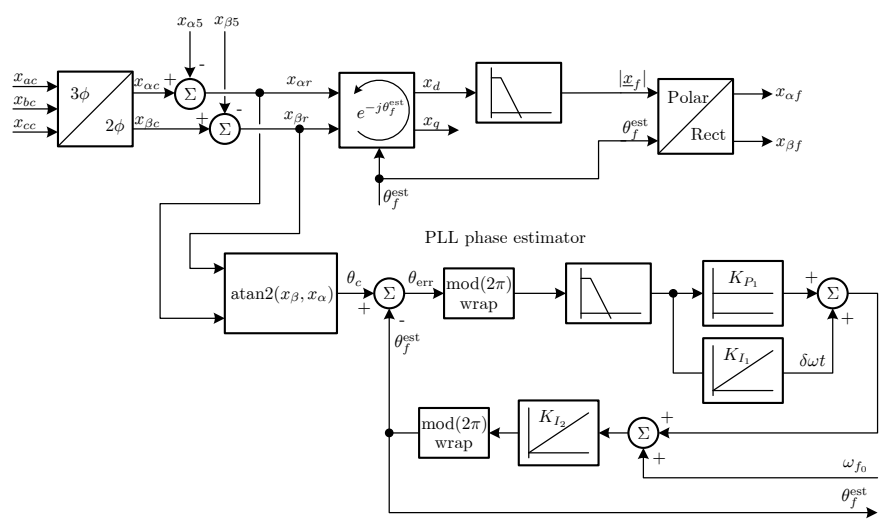

$5^{\text {TH }}$ HARMONIC

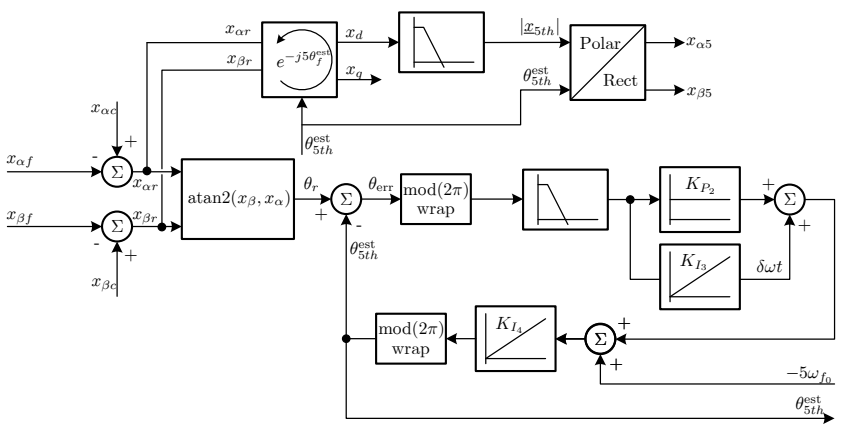

Figure 2: Block diagram of the two interconnected APLLs to extract the fundamental and the 5th harmonic.

of PLLs have been used in power systems research for a long time. After consideration of the EPLL, it was decided to go for our own three phase PLL approach using a simpler and, we believe, a more understandable PLL architecture.

The new PLL structure developed is called the Advanced Phase Locked Loop (APLL). Figure 2 is a block diagram of its structure. This diagram actually shows two APLLs since they are being used to extract the fundamental and the 5th harmonic from the input signal. Similar interconnection of the APLLs is used to extract more harmonics.

The key features of the APLL are that the phase loop is a linear loop, and when in lock $\theta_{\text {err }}$ is a DC value. Therefore there is no phase shift issues in the loop, as there are in more traditional PLL implementations based on the use of multiplier based phase detectors. Similarly, in the waveform estimation section phase shift in the low pass filter (LPF) does not result in phase shift in the waveforms. Secondly, there is a PI regulator in the phase feedback loop that allows the loop to achieve zero phase error even under conditions where the centre frequency of the PLL is not equal to the true frequency. Thirdly, the inputs to the loop have the harmonic estimates from the other loops subtracted from the initial composite waveform before going into the loop proper. Finally, the loop "naturally" allows very accurate forward prediction of the identified harmonic. These features when combined have resulted in excellent harmonic extraction performance.

\section{Simulation Results}

The simulation used is implemented in Saber, and is very accurate and comprehensive. The APLLs where added to an already existing simulation of a $\mathrm{H}$-StatCom, which completely simulates the H-bridge phase legs, the start-up sequencing, and all the control loops. It is a multi-mode simulation, with the control implemented digitally with the precise timing of the experimental H-StatCom at the University of Newcastle. The key control algorithms are implemented as ' $\mathrm{C}$ ' DLLs, and this code is used in the actual experimental system as well.

Let us firstly consider a couple of results showing the performance of the APLL itself. These tests were implemented in a separate program, and are comprehensively reported in another paper which compares the APLL performance with other approaches. The basic waveform used to test the APLL is of the form:

$$
\begin{aligned}
& x_{a}=\quad 100 \sin \left(\omega t+k_{1} \delta_{1}\right)+30 \sin \left(5 \omega t+k_{5} \delta_{5}\right)+15 \sin \left(7 \omega t+k_{7} \delta_{7}\right) \\
& x_{b}=\quad 100 \sin \left(\omega t-\frac{2 \pi}{3}-k_{1} \delta_{1}\right) \\
&+30 \sin \left[5\left(\omega t-\frac{2 \pi}{3}\right)-k_{5} \delta_{5}\right] \\
&+15 \sin \left[7\left(\omega t-\frac{2 \pi}{3}\right)-k_{7} \delta_{7}\right] \\
& x_{c}=100 \sin \left(\omega t+\frac{2 \pi}{3}-k_{1} \delta_{1}\right) \\
&+30 \sin \left[5\left(\omega t+\frac{2 \pi}{3}\right)-k_{5} \delta_{5}\right] \\
&+15 \sin \left[7\left(\omega t+\frac{2 \pi}{3}\right)-k_{7} \delta_{7}\right]
\end{aligned}
$$

where $\omega=307.88 \mathrm{rads} / \mathrm{sec}\left(49 \mathrm{~Hz}\right.$ supply frequency ${ }^{1}$ ). The PLLs in the simulation assume that the supply frequency is $50 \mathrm{~Hz}$. The simulation allows the basic waveforms to be modified with large amplitude glitches, and harmonic amplitude and phase variations. This is to test the tracking of the three schemes under such conditions. The glitch in the input waveform multiplies the composite waveform amplitude by 5 , and the glitch is there for 10 samples (i.e. $2 \mathrm{msec}$ ). There is an step amplitude change at the mid point (5000 samples) into the simulation. Only the amplitude of the harmonics have been changed by multiplying the 5th and 7th harmonics by 1.3 and 1.4 respectively. This is designed to test the performance of the fundamental harmonic estimation with respect to changes in the amplitudes of other harmonics, and also the transient harmonic amplitude estimation performance. The last transient input is a harmonic phase change. This is a relative phase change between the fundamental and its harmonics. The initial phase shift of the 5th harmonic is $\delta_{5}=\frac{\pi}{4} \mathrm{rads}$, and the 7 th

\footnotetext{
${ }^{1}$ This supply frequency is to simulate that the true supply frequency can be different from the nominal $50 \mathrm{~Hz}$.
} 


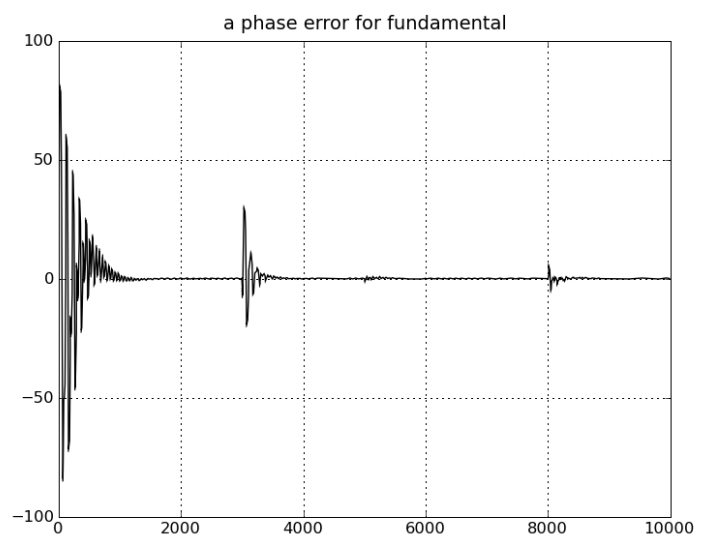

Figure 3: Error between the ' $a$ ' phase fundamental and the APLL estimated fundamental.

harmonic $\delta_{7}=\frac{\pi}{3}$ rads (note $\delta_{1}=0$ ). These initial phase offsets are then multiplied by $k_{5}=2.0$ and $k_{7}=2.5$ respectively at 8000 samples.

Figure 3 shows the error between the estimated fundamental of phase ' $a$ ' and the actual phase ' $a$ '. Note the increased error at the point of the disturbances. However the error quickly disappears, demonstrating the excellent performance of the APLL. The two plots shown in Figure 4 are similar plots for the 5th and 7th 'a' phase harmonic estimates. The disturbances are larger in this case, but again the error quickly damps out.

The H-StatCom test simulation parameters are that the load is absorbing a fundamental current of $75 \mathrm{Amp}$ peak, and there are two harmonics - the 5th at 15 Amp peak and the 7th at 10 Amp peak (similar to a diode rectifier load). The phase angle of the fundamental current is $50^{\circ}$ lagging. The H-StatCom switches in the active filtering algorithm at 0.6 seconds, and the VAR compensation at 0.7 seconds. The results are shown in Figure 5 on the next page ${ }^{2}$. As can be seen from the waveform plots the harmonics are highly suppressed, and the VAR compensation compensates for the inductive load so that the source current is moved to a phase angle of $0^{\circ}$. The transient performance of the algorithm is excellent. The second subplot shows the harmonics of the key currents. The active filtering suppresses the 5 th harmonic by $-29 \mathrm{~dB}$, and the 7 th harmonic by $-23.5 \mathrm{~dB}$.

The performance of the APLLs in tracking the harmonics is shown in the Figure 5(c). The stepped waveforms are the digital estimates of the harmonic currents. Notice that the estimates very accurately follow the harmonics one control interval ahead. The phase error is in the milli-radians range.

Figure $5 \mathrm{~d}$ shows the actual ' $\mathrm{a}$ ' phase H-StatCom output current and the source side current under a slightly different set of conditions. In this situation the fundamental VAR compensation is turned on at 0.7 secs, and the active filtering at 0.8 secs. One can see the after 0.7 secs the source side current

${ }^{2}$ Note that Figure $5 \mathrm{~d}$ is an exception, as this plot shows the actual $\mathrm{H}$ StatCom currents under slightly different conditions.

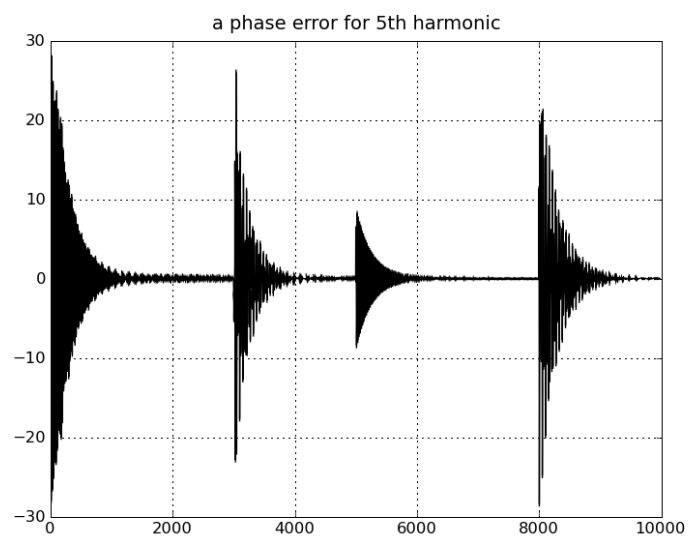

(a) APLL 5th harmonic

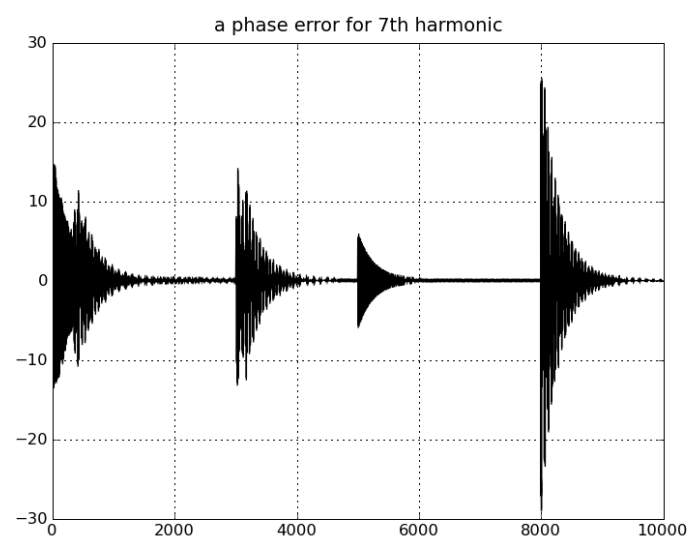

(b) APLL 7th harmonic

Figure 4: 5th harmonic and 7th harmonic errors for the APLL. Supply frequency $=49 \mathrm{~Hz}$, VCO frequency $=50 \mathrm{~Hz}$.

deceases considerably as the fundamental VARS are supplied by the H-StatCom. The a_phase_output_current plot of the H-StatCom output current can be seen to be sinusoidal between 0.7 secs and 0.8 secs. At 0.8 secs the active filtering is turned on and the source side current now becomes sinusoidal, and the H-StatCom current has harmonics as it is now supplying the harmonic currents. As with the previous results the excellent transient performance of the H-StatCom control is evident.

\section{CONCLUSIONS}

This paper presents a novel phase locked loop based filtering and prediction algorithm for harmonics. This algorithm is integrated with a novel dead-beat control strategy for $\mathrm{H}$ StatComs to implement a controller for active filtering and VAR compensation. Comprehensive simulation results show that the new strategy has excellent performance, with substantial reductions in the harmonics. These large reductions are achieved without the use of feedback because of the ability of 

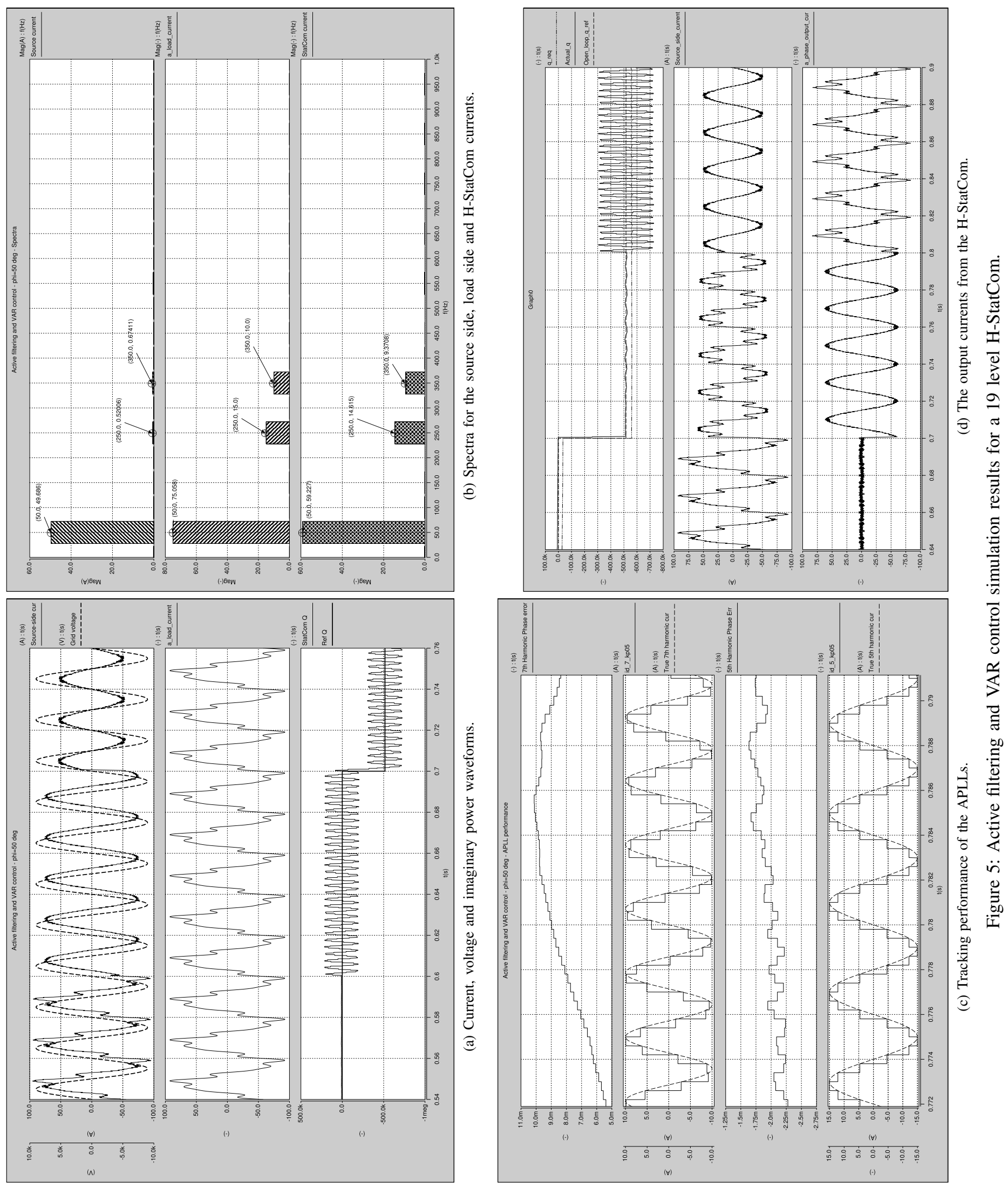
the APLL to provide very accurate predictions of the future harmonic currents. This virtually completely eliminates the effects of digital computational delay.

The APLL structure, from the testing that has been undertaken to date, appears to be offer robust and accurate estimation of harmonics, and has performance superior to other PLL approaches currently being used.

\section{REFERENCES}

[1] J. Ning and Y. He, "Phase-shifted suboptimal pulse-width modulation strategy for multilevel inverter," in Industrial Electronics and Applications, 2006 IST IEEE Conference on, (Singapore), pp. 1-5, May 2006

[2] Z. Du, L. M. Tolbert, and J. N. Chiasson, "Active harmonic elimination for multilevel converters," IEEE Trans. Power Electron., vol. 21, pp. 459-469, Mar. 2006.

[3] Q. Song, W. Liu, and Z. Yuan, "Multilevel optimal modulation and dynamic control strategies for STATCOMs using cascaded multilevel inverters," IEEE Trans. Power Del., vol. 22, pp. 1937-1946, July 2007.

[4] H. Akagi, Y. Kanazawa, and A. Nabae, "Instantaneous reactive power compensators comprising switching devices without energy storage components," IEEE Transaction on Industry Applications, vol. 20, pp. 625630, May/June 1984.

[5] H. Akagi, E. H. Watanabe, and M. Aredes, Instantaneous Power Theory and Application to Power Conditioning. John Wiley and Sons, 2007.

[6] D. G. Holmes and D. A. Martin, "Implementation of a direct digital predictive current controller for single and three phase voltage source inverters," in Industry Applications Conference, 1996. Thirty-First IAS Annual Meeting, IAS '96., Conference Record of the 1996 IEEE, vol. 2, (San Diego, CA), pp. 906-913, Oct. 1996.

[7] R. Betz, B. Cook, and S. Henriksen, "A digital current controller for three phase voltage source inverters," Conference Record of the IEEEIAS Annual Meeting, pp. 722-729, New Orleans, Oct. 1997.

[8] R. E. Betz, T. Summers, and B. Cook., "Outline of the control design for a cascaded h-bridge statcom," in Proceedings of the IEEE-IAS Annual Meeting, Edmonton Canada, Oct 2008. ISBN 978-1-4244-2279-1.

[9] H. Akagi, S. Inoue, and T. Yoshii, "Control and performance of a transformerless cascade PWM STATCOM with star configuration," IEEE Trans. Ind. Appl., vol. 43, pp. 1041-1049, July/Aug. 2007.

[10] M. J. Newman, D. G. Holmes, J. G. Nielsen, and F. Blaabjerg, "A Dynamic Voltage Restorer (DVR) with Selective Harmonic Compensation and Medium Voltage Level," in Proceedings of the IEEE-IAS Annual Meeting, October 2003.

[11] M. Karimi-Ghartemani and M. R. Iravani, "A new phase-locked loop (PLL) system," in Circuits and Systems, 2001. MWSCAS 2001. Proceedings of the 44th IEEE 2001 Midwest Symposium on, vol. 1, (Dayton, OH), pp. 421-424, Aug. 2001. 